\title{
Plasma membrane-bound AGC3 kinases phosphorylate PIN auxin carriers at TPRXS(N/S) motifs to direct apical PIN recycling
}

\author{
Pankaj Dhonukshe ${ }^{1, *, \pi}$, Fang Huang ${ }^{2, \#, *}$, Carlos S. Galvan-Ampudia ${ }^{2, \$, *}$, Ari Pekka Mähönen ${ }^{1, \dagger}$,

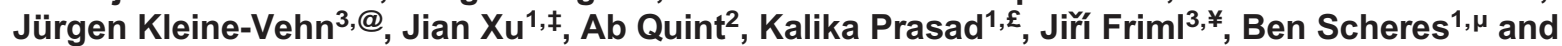 \\ Remko Offringa ${ }^{2, \S}$ \\ ${ }^{1}$ Section of Molecular Genetics, Department of Biology, Utrecht University, Padualaan 8, 3584CH Utrecht, The Netherlands. \\ ${ }^{2}$ Molecular and Developmental Genetics, Institute Biology Leiden, Leiden University, Sylvius Laboratory, Sylviusweg 72, $2333 B E$ Leiden, The Netherlands. \\ ${ }^{3}$ Department of Plant Systems Biology, Flanders Institute for Biotechnology, Technologiepark 927, 9052 Ghent, Belgium. \\ "These authors contributed equally to this work \\ "No longer at this address \\ \#Present address: Institute of Integrative Biology, University of Liverpool, Crown Street, Liverpool L69 7ZB, UK. \\ \$Present address: Plant Reproduction and Development, Ens de Lyon, 15 parvis René Descartes - BP 700069342 Lyon Cedex 07 , France. \\ †Present address: Institute of Biotechnology, University of Helsinki, P.O. Box 65, FIN-00014, Helsinki, Finland. \\ ${ }^{@}$ Present address: Department of Applied Genetics and Cell Biology (DAGZ), Muthgasse 18, 1190 Wien, Austria. \\ †Present address: Department of Biological Sciences, Faculty of Sciences, National University of Singapore, 14 Science Drive 4 , Singapore 117543. \\ EPresent address: School of Biology, Indian Institute of Science Education and Research, Thiruvananthapuram 695016, Kerala, India. \\ ¥Present address: Institute of Science and Technology Austria (IST Austria), Am Campus 1, A-3400 Klosterneuburg, Austria. \\ "Present address: Plant Developmental Biology, Wageningen University, Postbus 633, 6700AP Wageningen, The Netherlands \\ §Author for correspondence (r.offringa@biology.leidenuniv.nl)
}

The authors informed us of problems related to Fig. 3C and Fig. 7D in Development 137, 3245-3255. Both issues were noted by an investigation by the Technical Committee of Utrecht University (UTC) into this publication. Based on the findings provided (detailed below), the journal has decided that the major conclusions of the paper are not affected and that retraction is not required, but that a correction should be provided with an explanation of the circumstances. This course of action complies with our policy on correction of issues in the scientific record, which states: "Should an error appear in a published article that affects scientific meaning or author credibility but does not affect the overall results and conclusions of the paper, our policy is to publish a Correction".

For Fig. 3C, the UTC concluded that it was the result of "cut-and-paste", which is "not indicated by a solid line, nor is it mentioned in the figure legend, as prescribed by journal policy". Fig. 3C presents results of in vitro phosphorylation of the different PIN2 hydrophilic loop (PIN2 HL) versions by the kinases PID, WAG1 and WAG2. The results were more or less similar for the three kinases (Fig. 3 revised version), and in order to reduce the figure size, it was decided that it would be sufficient to show the full data for the PINOID kinase, and to focus on the phosphorylation results for the wild-type version and the loss-of-phosphorylation version of the PIN2 HL for the WAG1 and WAG2 kinases. This involved splicing, and although the splicing is clearly visible in the original Fig. 3C, the appropriate presentation according to the standards of the journal would have been to leave space between the spliced parts. As demonstrated by the comparison between the revised Fig. 3 (below) and the original Fig. 3C, the spliced version represents part of the original data. In the revised version of Fig. 3 the full data set is shown and a more detailed description of the results is provided in the revised figure legend text.

With respect to Fig. 7D, the UTC noted that "it is not possible that random background noises, taken from two images, are identical. The most likely explanation is that the same green panel was used for the left and middle panel of Fig. 7D". The original data were not available to the UTC, and this anomaly could not be resolved. Development also appointed its own independent expert to analyse the images, who concluded that "the two images are almost identical (except for the region purporting to show photoconversion) and it is highly unlikely that these could represent different time points. The analysis suggests that one of these images appears to have been generated by manipulating the other." For this reason the authors and editors have seriously considered retraction of Development 137, 3245-3255. However, the thorough analysis by the UTC and the Development editors identified no other abnormalities in the data. The authors and editors therefore feel that removing Fig. 7D and the related text from the publication provides the optimal solution for protecting the integrity of the scientific record.

According to the authors: "The main conclusion of the paper is that the AGC3 kinases PID, WAG1 and WAG2, and not other plant AGC kinases, instruct recruitment of PINs into the GNOM-independent apical recycling pathway by phosphorylating the middle serine in three conserved TPRXS(N/S) motifs. Based on data provided in Development 137, 3245-3255 and also in other publications (Friml et al., 2004, Science 306, 862-865; Michniewicz et al., 2007, Cell 130, 1044-1056; Kleine-Vehn et al., 2008, Curr. Biol. 18, 1-6; Kleine-Vehn et al., 2009, Plant Cell 21, 3839-3849; Huang et al., 2010, Plant Cell 22,1129-1142), a model is proposed by which apolarly localized kinases phosphorylate PINs at the plasma membrane after PIN secretion, and trigger endocytosis-dependent PIN transcytosis (the trafficking of existing PIN cargo from one polar domain to another) to the apical domain. The objective of the photoconversion and time-lapse imaging experiments presented in Fig. 7D was merely to provide evidence for phosphorylation-triggered PIN transcytosis. PIN transcytosis was reported before by Kleine-Vehn et al. in 2008, Curr. Biol. 18, 1-6; and in 2009, Plant Cell 21, 3839-3849, and a role for PID in this process was also proposed, but not definitively demonstrated, in that latter paper." Omission of Fig. 7D does not affect the main conclusions, and given that phosphorylation-triggered PIN transcytosis is only one minor aspect of the full paper, the editorial board of Development has 
A .... GAKLLISEQF PETAGSITSF RVDSDVISLN GREPLQTDAE IGDDGKLHVV VRRSSAASSM ISSFNKSHGG
GLNSSMITPR A $\mathbf{1}$ SNLTGVEIY SVQSSREPTP RA $\mathbf{2}$ SFNQTDF YAMFNASKAP SPRHGYTNSY GGAGAGPGGD VYSLQSSKGV 3 TPRTSNFDEE VMKTAKKAGR $\ldots \ldots \ldots$
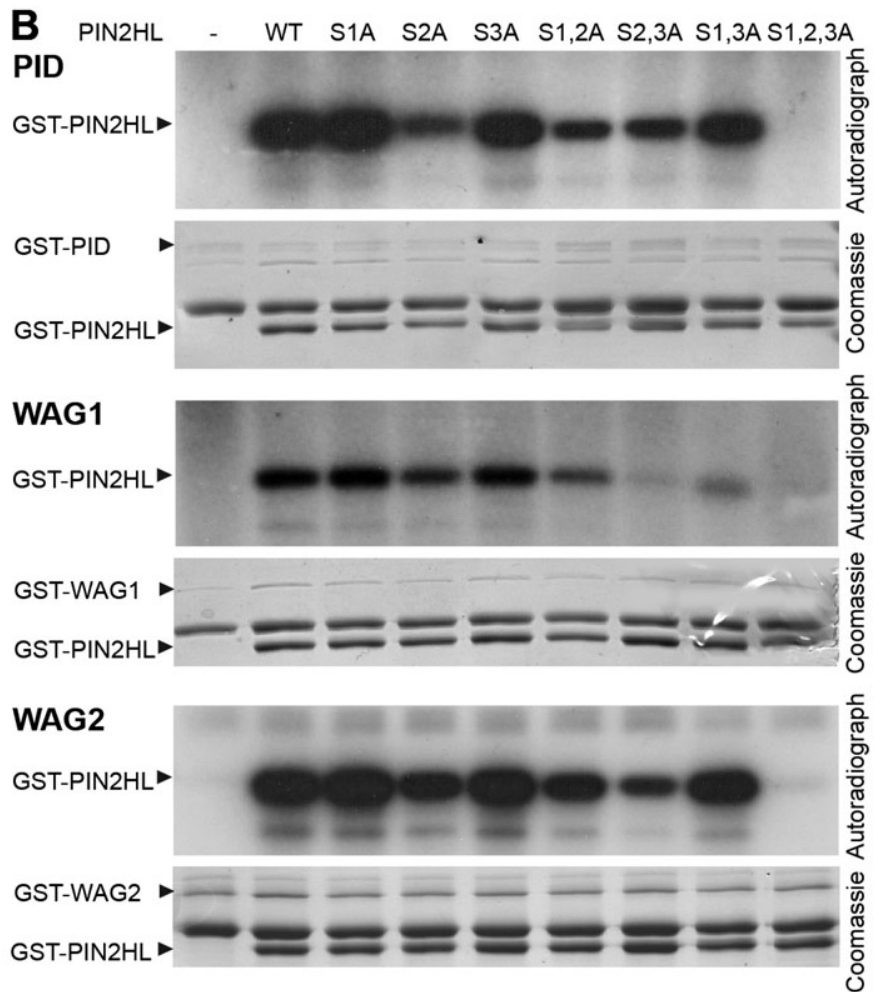

Fig. 3 (Revised). The central serines in three conserved TPRXS(N/S) motifs of the PIN2HL are the phosphorylation targets of PID, WAG1 and WAG2 kinases in vitro. (A) N-terminal part of the PIN2HL with the phosphoserines in the three TPRXS(N/S) motifs indicated. (B) GST-PIN2HL is phosphorylated by GST-PID and GST-WAG2, and also by GST-WAG1, albeit at lower levels. The results suggest that there are differences in the kinase activities and the contribution of the individual serines (S1, S2 and S3) in the phosphorylation of the PIN2HL by the kinases (e.g. replacing S2 for alanine seems to give the strongest reduction, whereas replacing S3 only leads to a significant reduction when combined with other substitutions), but the overall trend is that the phosphorylation is gradually reduced when one or two serines are replaced with alanines (indicated as S1A, S2A, S3A, S1,2A $\mathrm{S} 2,3 \mathrm{~A}$ and $\mathrm{S} 1,3 \mathrm{~A}$, respectively) and is completely abolished when all of the three serines are mutated to alanines $(\mathrm{S} 1,2,3 \mathrm{~A})$.

decided that a correction suffices. The authors agree with this decision. The readers of Development 137, 3245-3255 are therefore advised to ignore the data presented in Fig. 7D and the related text:

P. 3251: "Interestingly, upon reversal of PID induction (by washing out the induction medium), apically localized PIN2::PIN1-EosFP2 returned to the basal PM domain (Fig. 7D), indicating that constitutive PID action is required for maintaining apical PIN localization." P. 3252, legend Fig. 7: "Green-to-red photoconverted PIN2::PIN1-EosFP2 displays apical-to-basal relocation after estradiol washout (D)."

Pankaj Dhonukshe, the co-first and co-corresponding author of the original manuscript, is not listed as an author on this Correction upon his request. He provided us with the following statement, expressing his opinion:

"Pankaj Dhonukshe does not agree with the content of the Correction Notice as:

i. the anomaly in the first green panel (before photoconversion) at Fig. 7D does not affect the published conclusion drawn from research findings. The conclusion is solely based and derived from separately acquired red panels shown in Fig. 7D. Deriving conclusions from only red panels is a standard practice.

ii. The anomaly at Fig. 7D does not affect the published conclusion. The current content related to Fig. 7D should therefore be revised, and not removed.

iii. For Fig. 3C, original data did not accurately support the published conclusion. As per the Correction Notice, original data not previously shown is included and new conclusions are derived from this data. Readers should be aware that original conclusions are therefore not accurately supported."

We note that the affiliations of several authors have been updated in the author list above. 